\title{
Evidence for hybridisation in the Tynong Province granitoids, Lachlan Fold Belt, eastern Australia
}

\author{
K. R. Regmi ${ }^{\mathrm{a}, \mathrm{b}}$, R. F. Weinberg ${ }^{\mathrm{a} *}$, I. A. Nicholls ${ }^{\mathrm{a}}$, R. Maas ${ }^{\mathrm{c}}$, and M. Raveggi ${ }^{\mathrm{a}}$ \\ ${ }^{a}$ School of Earth, Atmosphere and Environment, Monash University, Clayton, VIC 3800, \\ Australia \\ ${ }^{\mathrm{b}}$ Department of Geology, Faculty of Science, University of Namibia, Windhoek, Namibia \\ ${ }^{\mathrm{c}}$ School of Earth Sciences, University of Melbourne, VIC 3010, Australia \\ *Corresponding author, e-mail address: Roberto.weinberg@monash.edu, tel: +61-3- \\ 99054902
}

\section{SUPPLEMENTARY PAPERS}

Australian Journal of Earth Sciences (2016) 63, http://dx.doi.org/10.1080/08120099.2016.1180321

Copies of Supplementary Papers may be obtained from the Geological Society of Australia's website (www.gsa.org.au), the Australian Journal of Earth Sciences website (www.ajes.com.au) or from the National Library of Australia's Pandora archive (http://nla.gov.au/nla.arc-25194).

\section{SUPPLEMENTARY PAPERS}

Table $1 \mathrm{U}-\mathrm{Pb}$ isotope data for the Toorongo pluton (sample 406).

Table $2 \mathrm{U}-\mathrm{Pb}$ isotope data for the Tynong pluton (sample TYN5).

Table 3 Major and trace element data for the Tynong Province granitoids.

Table $4 \mathrm{Lu}$ and $\mathrm{Hf}$ isotopic data for zircons from one sample of the Toorongo (sample 406, $37^{\circ} 48^{\prime} 41^{\prime \prime} \mathrm{S}, 146^{\circ} 05^{\prime} 40^{\prime \prime} \mathrm{E}$ ), and two samples from the Tynong pluton (samples 2803-8 and TYN5, 38 $03^{\prime} 30^{\prime \prime}$ 'S, $145^{\circ} 37^{\prime} 26^{\prime \prime}$ ), Lachlan Orogen, eastern Australia. 
Table $1 \mathrm{U}-\mathrm{Pb}$ isotope data for the Toorongo pluton (sample 406).

\begin{tabular}{|c|c|c|c|c|c|c|c|c|c|}
\hline & ${ }^{238} \mathrm{U} /{ }^{206} \mathrm{~Pb}$ & $2 \mathrm{~s}$ & ${ }^{207} \mathrm{~Pb} /{ }^{206} \mathrm{~Pb}$ & $2 s$ & $\begin{array}{l}{ }^{238} \mathrm{U} /{ }^{206} \mathrm{~Pb} \\
\text { ages (Ma) }\end{array}$ & $2 \mathrm{~s}$ & $\begin{array}{c}{ }^{207} \mathrm{~Pb} \\
\text { corrected } \\
\text { ages }(\mathrm{Ma})\end{array}$ & $2 s^{*}$ & Comment \\
\hline KR09020406-2r & 17.1409 & 0.3232 & 0.0556 & 0.0020 & 366 & 13 & 365 & 22 & OK \\
\hline KR09020406-3 & 16.1629 & 0.3083 & 0.0549 & 0.0023 & 387 & 14 & 387 & 23 & OK \\
\hline KR09020406-3c & 16.0308 & 0.3803 & 0.0567 & 0.0041 & 390 & 18 & 389 & 25 & OK \\
\hline KR09020406-4 & 16.5371 & 0.3063 & 0.0530 & 0.0019 & 378 & 13 & 379 & 22 & OK \\
\hline KR09020406-4-2 & 16.8294 & 0.3172 & 0.0545 & 0.0020 & 372 & 13 & 372 & 22 & OK \\
\hline KR09020406-5 & 17.3641 & 0.4221 & 0.0544 & 0.0037 & 361 & 17 & 361 & 24 & OK \\
\hline KR09020406-6r & 16.1943 & 0.3095 & 0.0542 & 0.0021 & 386 & 14 & 386 & 23 & OK \\
\hline KR09020406-6c & 16.3586 & 0.3158 & 0.0632 & 0.0026 & 382 & 14 & 378 & 23 & OK \\
\hline KR09020406-7r & 16.4177 & 0.3181 & 0.0612 & 0.0025 & 381 & 14 & 378 & 23 & OK \\
\hline KR09020406-8c & 16.3345 & 0.3042 & 0.0597 & 0.0021 & 383 & 14 & 381 & 23 & OK \\
\hline KR09020406-8r & 17.4064 & 0.3272 & 0.0548 & 0.0019 & 360 & 13 & 360 & 21 & OK \\
\hline KR09020406-9r & 16.1499 & 0.3182 & 0.0524 & 0.0025 & 387 & 15 & 388 & 23 & OK \\
\hline KR09020406-10c & 16.0282 & 0.3340 & 0.0564 & 0.0026 & 390 & 15 & 389 & 24 & OK \\
\hline KR09020406-11r & 17.6772 & 0.3375 & 0.0611 & 0.0024 & 355 & 13 & 352 & 21 & OK \\
\hline KR09020406-11c & 17.5039 & 0.3860 & 0.0664 & 0.0033 & 358 & 15 & 353 & 23 & OK \\
\hline KR09020406-12r & 17.0068 & 0.3297 & 0.0540 & 0.0021 & 368 & 14 & 368 & 22 & OK \\
\hline KR09020406-12c & 16.5453 & 0.3778 & 0.0549 & 0.0034 & 378 & 16 & 378 & 24 & OK \\
\hline KR09020406-13r & 17.2147 & 0.3615 & 0.0575 & 0.0028 & 364 & 15 & 362 & 22 & OK \\
\hline KR09020406-14 & 16.7588 & 0.3820 & 0.0535 & 0.0029 & 374 & 16 & 374 & 24 & OK \\
\hline KR09020406-15r & 16.5536 & 0.3343 & 0.0595 & 0.0027 & 378 & 15 & 376 & 23 & OK \\
\hline KR09020406-15c & 15.9566 & 0.3514 & 0.0540 & 0.0031 & 392 & 16 & 392 & 25 & OK \\
\hline KR09020406-16 & 17.0707 & 0.3613 & 0.0630 & 0.0027 & 367 & 15 & 363 & 23 & OK \\
\hline KR09020406-17r & 17.4948 & 0.3367 & 0.0526 & 0.0020 & 358 & 13 & 359 & 21 & OK \\
\hline KR09020406-17c & 17.3943 & 0.3752 & 0.0526 & 0.0025 & 360 & 15 & 361 & 22 & OK \\
\hline KR09020406-18r & 16.7701 & 0.3656 & 0.0627 & 0.0029 & 373 & 15 & 369 & 23 & OK \\
\hline KR09020406-19-2 & 17.6616 & 0.3806 & 0.0584 & 0.0030 & 355 & 15 & 353 & 22 & OK \\
\hline KR09020406-20 & 16.7112 & 0.3351 & 0.0562 & 0.0025 & 375 & 14 & 374 & 23 & OK \\
\hline KR09020406-25 & $\underline{14.7254}$ & $\underline{0.2992}$ & $\underline{0.0604}$ & $\underline{0.0028}$ & $\underline{424}$ & $\underline{16}$ & $\underline{421}$ & $\underline{26}$ & $\begin{array}{l}\text { Older age, possible } \\
\text { inheritance }\end{array}$ \\
\hline
\end{tabular}




\begin{tabular}{|c|c|c|c|c|c|c|c|c|c|}
\hline & ${ }^{238} \mathrm{U} /{ }^{206} \mathrm{~Pb}$ & $2 \mathrm{~s}$ & ${ }^{207} \mathrm{~Pb} /{ }^{206} \mathrm{~Pb}$ & $2 \mathrm{~s}$ & $\begin{array}{l}{ }^{238} \mathrm{U} /{ }^{206} \mathrm{~Pb} \\
\text { ages (Ma) }\end{array}$ & $2 \mathrm{~s}$ & $\begin{array}{c}{ }^{207} \mathrm{~Pb} \\
\text { corrected } \\
\text { ages }(\mathrm{Ma})\end{array}$ & $2 s^{*}$ & Comment \\
\hline KR09020406-26 & 16.8209 & 0.3339 & 0.0543 & 0.0025 & 372 & 14 & 372 & 22 & OK \\
\hline KR09020406-30 & $\underline{12.1551}$ & 0.2275 & $\underline{0.0570}$ & 0.0017 & $\underline{510}$ & $\underline{18}$ & $\underline{510}$ & $\underline{30}$ & $\begin{array}{l}\text { Older age, possible } \\
\text { inheritance }\end{array}$ \\
\hline KR09020406-31r & 18.0636 & 0.3524 & 0.0559 & 0.0021 & 347 & 13 & 346 & 21 & $\overline{\mathrm{OK}}$ \\
\hline KR09020406-32 & 17.1880 & 0.3486 & 0.0548 & 0.0023 & 365 & 14 & 364 & 22 & OK \\
\hline KR09020406-33 & 17.2325 & 0.3801 & 0.0582 & 0.0028 & 364 & 15 & 362 & 23 & OK \\
\hline KR09020406-34r & 16.5180 & 0.3220 & 0.0572 & 0.0019 & 379 & 14 & 378 & 23 & OK \\
\hline KR09020406-35c & 16.2866 & 0.3130 & 0.0548 & 0.0020 & 384 & 14 & 384 & 23 & OK \\
\hline KR09020406-35r & 16.6445 & 0.3269 & 0.0546 & 0.0020 & 376 & 14 & 376 & 23 & OK \\
\hline$\underline{K R 09020406-36}$ & $\underline{15.4392}$ & $\underline{0.3146}$ & $\underline{0.0604}$ & $\underline{0.0026}$ & $\underline{405}$ & $\underline{16}$ & $\underline{402}$ & $\underline{25}$ & $\begin{array}{l}\text { Older age, possible } \\
\text { inheritance }\end{array}$ \\
\hline KR09020406-37 & 17.2028 & 0.3788 & 0.0557 & 0.0030 & 364 & 15 & 363 & 23 & $\overline{\mathrm{OK}}$ \\
\hline KR09020406-39 & 16.3827 & 0.3113 & 0.0549 & 0.0020 & 382 & 14 & 382 & 23 & OK \\
\hline KR09020406-41r & 16.7029 & 0.3180 & 0.0517 & 0.0019 & 375 & 14 & 376 & 22 & OK \\
\hline KR09020406-42 & 16.2602 & 0.3226 & 0.0525 & 0.0023 & 385 & 15 & 386 & 23 & OK \\
\hline KR09020406-43r & 17.3551 & 0.3313 & 0.0518 & 0.0019 & 361 & 13 & 362 & 21 & OK \\
\hline KR09020406-43c & 14.9633 & $\underline{0.3045}$ & $\underline{0.0585}$ & $\underline{0.0023}$ & $\underline{417}$ & $\underline{16}$ & $\underline{415}$ & $\underline{25}$ & $\begin{array}{l}\text { Older age, possible } \\
\text { inheritance }\end{array}$ \\
\hline KR09020406-44r & 16.8862 & 0.3194 & 0.0527 & 0.0018 & 371 & 13 & 371 & 22 & $\mathrm{OK}$ \\
\hline KR09020406-44c & 16.2760 & 0.3179 & 0.0528 & 0.0020 & 384 & 14 & 385 & 23 & OK \\
\hline KR09020406-45 & 16.4123 & 0.3178 & 0.0522 & 0.0021 & 381 & 14 & 382 & 23 & OK \\
\hline
\end{tabular}

$2 \mathrm{~s}^{*}$ is the cumulative error reported as the square root of the sum of the squares of the internal precision and external precision $\left(4.7 \%\right.$ on the ${ }^{238} \mathrm{U} /{ }^{206} \mathrm{~Pb}$ of the standard 91500$)$. Analyses in italics and underlined were not used in age calculations, notice that four of them are interpreted to represent inheritance ( $>400$ Ma). 
Table $2 \mathrm{U}-\mathrm{Pb}$ isotope data for the Tynong pluton (sample TYN5).

\begin{tabular}{|c|c|c|c|c|c|c|c|c|c|}
\hline & ${ }^{238} \mathrm{U} /{ }^{206} \mathrm{~Pb}$ & $2 s$ & ${ }^{207} \mathrm{~Pb} /{ }^{206} \mathrm{~Pb}$ & $2 s$ & $\begin{array}{l}{ }^{238} \mathrm{U} /{ }^{206} \mathrm{~Pb} \\
\text { ages (Ma) }\end{array}$ & $2 s$ & $\begin{array}{l}{ }^{207} \mathrm{~Pb} \\
\text { corrected } \\
\text { ages }(\mathrm{Ma})\end{array}$ & $2 s^{*}$ & Comment \\
\hline Tyn5-1C & $\underline{20.0844}$ & 0.5002 & $\underline{0.0578}$ & 0.0041 & $\underline{313}$ & $\underline{8}$ & $\underline{311}$ & $\underline{16}$ & $\underline{P b \text { loss? }}$ \\
\hline Tyn5-2 & 18.1159 & 0.4201 & 0.0716 & 0.0038 & 346 & 8 & 339 & 18 & OK \\
\hline Tyn5-3r & 18.0310 & 0.5072 & 0.0636 & 0.0058 & 348 & 10 & 344 & 19 & OK \\
\hline Tyn5-3c & 21.2993 & $\underline{0.6442}$ & $\underline{0.1082}$ & $\underline{0.0088}$ & $\underline{296}$ & $\underline{9}$ & $\underline{274}$ & $\underline{15}$ & Discordant/Common Pb \\
\hline Tyn5-4c & $\underline{16.3666}$ & $\underline{0.5572}$ & $\underline{0.0985}$ & $\underline{0.0099}$ & 382 & $\underline{13}$ & $\underline{362}$ & $\underline{21}$ & Discordant/Common Pb \\
\hline Tyn5-4r & 16.8776 & 0.4273 & 0.0663 & 0.0043 & 371 & 9 & 365 & 19 & OK \\
\hline Tyn5-5 & 17.7683 & 0.4420 & 0.0573 & 0.0038 & 353 & 9 & 351 & 19 & OK \\
\hline Tyn5-6 & 14.7536 & $\underline{0.4832}$ & $\underline{0.1249}$ & 0.0122 & $\underline{423}$ & $\underline{13}$ & $\underline{387}$ & $\underline{23}$ & Discordant/Common Pb \\
\hline Tyn5-7r & 15.9795 & 0.3881 & 0.0772 & 0.0046 & 391 & 9 & 380 & 20 & OK \\
\hline Tyn5-7c & 16.6973 & 0.4405 & 0.0582 & 0.0043 & 375 & 10 & 373 & 20 & OK \\
\hline Tyn5-8 & $\underline{16.4312}$ & $\underline{0.6048}$ & $\underline{0.1333}$ & $\underline{0.0143}$ & $\underline{381}$ & $\underline{14}$ & $\underline{344}$ & $\underline{21}$ & Discordant/Common Pb \\
\hline Tyn5-9 & 15.8028 & 0.5494 & 0.0566 & 0.0064 & 396 & 13 & 395 & 23 & OK \\
\hline Tyn5-10 & 15.3421 & 0.3813 & 0.0885 & 0.0052 & 407 & 10 & 390 & 21 & OK \\
\hline Tyn5-11 & 15.9744 & 0.4083 & 0.0593 & 0.0037 & 391 & 10 & 389 & 21 & OK \\
\hline Tyn5-12 & 17.7620 & 0.5553 & 0.0902 & 0.0077 & 353 & 11 & 337 & 19 & OK \\
\hline Tyn5-13 & 16.9119 & 1.1097 & 0.0723 & 0.0165 & 370 & 24 & 362 & 29 & Short acquisition but OK \\
\hline Tyn5-14 & 17.4398 & 0.4136 & 0.0649 & 0.0036 & 359 & 8 & 354 & 19 & OK \\
\hline Tyn5-15 & 17.0474 & 0.6277 & 0.0525 & 0.0063 & 367 & 13 & 368 & 22 & OK \\
\hline Tyn5-16 & 17.3943 & 0.6112 & 0.0546 & 0.0062 & 360 & 12 & 360 & 21 & OK \\
\hline Tyn5-17 & $\underline{16.4799}$ & $\underline{0.4889}$ & $\underline{0.1403}$ & $\underline{0.0106}$ & $\underline{380}$ & $\underline{11}$ & $\underline{340}$ & $\underline{19}$ & Discordant/Common Pb \\
\hline Tyn5-18 & 16.2813 & 0.4612 & 0.0728 & 0.0056 & 384 & 11 & 376 & 21 & OK \\
\hline Tyn5-19 & 16.8606 & 0.4662 & 0.0900 & 0.0067 & 371 & 10 & 355 & 19 & OK \\
\hline Tyn5-20r & 17.4581 & 0.8168 & 0.0625 & 0.0104 & 359 & 16 & 355 & 23 & OK \\
\hline Tyn5-20c & 16.4962 & 0.5606 & 0.0539 & 0.0057 & 379 & 13 & 380 & 22 & OK \\
\hline Tyn5-21 & 17.1733 & 0.6724 & 0.0909 & 0.0113 & 365 & 14 & 348 & 21 & OK \\
\hline Tyn5-22 & 16.8322 & 0.5213 & 0.0932 & 0.0085 & 372 & 11 & 354 & 20 & OK \\
\hline
\end{tabular}

$2 \mathrm{~s}^{*}$ is the cumulative error reported as the square root of the sum of the squares of the internal precision and external precision $(4.7 \%$ on the $238 \mathrm{U} / 206 \mathrm{~Pb}$ of the standard 91500). Analyses in italics and underlined were not used in age calculations. 
Table 3 Major and trace element data for the Tynong Province granitoids.

\begin{tabular}{|c|c|c|c|c|c|c|c|c|c|}
\hline $\begin{array}{c}\text { Pluton } \\
\text { Rock type }\end{array}$ & $\begin{array}{l}\text { Lysterfield } \\
\text { Aplytic } \\
\text { dyke }\end{array}$ & $\begin{array}{l}\text { Lysterfield } \\
\text { Granodiorite }\end{array}$ & $\begin{array}{l}\text { Lysterfield } \\
\text { Granodiorite }\end{array}$ & $\begin{array}{l}\text { Lysterfield } \\
\text { Granodiorite }\end{array}$ & $\begin{array}{l}\text { Lysterfield } \\
\text { Granodiorite }\end{array}$ & $\begin{array}{l}\text { Lysterfield } \\
\text { Enclave }\end{array}$ & $\begin{array}{l}\text { Lysterfield } \\
\text { Enclave }\end{array}$ & $\begin{array}{l}\text { Lysterfield } \\
\text { Enclave }\end{array}$ & $\begin{array}{l}\text { Lysterfield } \\
\text { Enclave }\end{array}$ \\
\hline Sample No & $1702-2$ & LYS1A & LYS3 & LYS4 & $1702-1$ & $1702-3 \mathrm{~A}$ & 1702-3B & $1702-4$ & 1702R-5 \\
\hline $\mathrm{SiO}_{2}$ & 76.99 & 65.19 & 67.99 & 63.83 & 67.09 & 68.48 & 55.07 & 54.23 & 59.87 \\
\hline $\mathrm{TiO}_{2}$ & 0.07 & 0.8 & 0.57 & 0.85 & 0.6 & 0.59 & 1.55 & 1.35 & 1 \\
\hline $\mathrm{Al}_{2} \mathrm{O}_{3}$ & 12.56 & 15.11 & 14.72 & 15.68 & 14.68 & 14.46 & 16.12 & 17.05 & 16.05 \\
\hline $\mathrm{Fe}_{2} \mathrm{O}_{3}$ & 0.57 & 4.69 & 3.65 & 5.2 & 4.05 & 3.8 & 8.38 & 8.69 & 7.18 \\
\hline $\mathrm{MnO}$ & 0.01 & 0.06 & 0.06 & 0.07 & 0.07 & 0.06 & 0.15 & 0.15 & 0.15 \\
\hline $\mathrm{MgO}$ & 0.26 & 2.19 & 1.94 & 2.69 & 2.09 & 1.94 & 4.88 & 4.81 & 4.18 \\
\hline $\mathrm{CaO}$ & 0.59 & 3.5 & 3.37 & 4.24 & 3.55 & 3.31 & 5.71 & 5.43 & 5.6 \\
\hline $\mathrm{Na}_{2} \mathrm{O}$ & 2.8 & 2.73 & 3.43 & 3.2 & 2.99 & 3 & 3.44 & 3.57 & 3.58 \\
\hline $\mathrm{K}_{2} \mathrm{O}$ & 5.55 & 4.25 & 3.52 & 3.13 & 3.33 & 3.41 & 2.96 & 2.97 & 1.77 \\
\hline $\mathrm{P}_{2} \mathrm{O}_{5}$ & bd & 0.26 & 0.14 & 0.24 & 0.14 & 0.13 & 0.34 & 0.4 & 0.19 \\
\hline L.O.I. & 0.21 & 0.82 & 0.6 & 0.74 & 0.52 & 0.42 & 0.7 & 0.52 & 0.83 \\
\hline Total & 99.6 & 99.64 & 100 & 99.9 & 99.09 & 99.59 & 99.29 & 99.16 & 100.38 \\
\hline ASI & 1.073 & 0.976 & 0.944 & 0.957 & 0.979 & 0.986 & 0.836 & 0.898 & 0.891 \\
\hline $\mathrm{Li}$ & & 37.2 & 33.5 & 32.0 & & & & & \\
\hline $\mathrm{Be}$ & & 3.7 & 2.5 & 3.2 & & & & & \\
\hline Sc & & 11.8 & 9.1 & 12.5 & 10.0 & 8.2 & 17.3 & 16.4 & 16.1 \\
\hline V & & 110.1 & 75.8 & 117.1 & 82 & 72 & 162 & 119 & 134 \\
\hline $\mathrm{Cr}$ & & 74.3 & 48.7 & 79.0 & 79 & 61 & 138 & 140 & 122 \\
\hline Co & & 193.5 & 85.4 & 85.7 & 23 & 33 & 35 & 32 & 24 \\
\hline $\mathrm{Ni}$ & & 34.6 & 35.6 & 44.3 & 39 & 35 & 83 & 85 & 97 \\
\hline $\mathrm{Cu}$ & & 37.8 & 32.7 & 49.6 & 38 & 38 & 314 & 352 & 379 \\
\hline $\mathrm{Zn}$ & & 65.9 & 49.2 & 69.0 & 45 & 43 & 89 & 102 & 82 \\
\hline $\mathrm{Ga}$ & & 18.0 & 16.1 & 18.8 & 16.2 & 15.6 & 18.3 & 21 & 17.8 \\
\hline ASI & & 1.0 & 1.5 & 1.1 & & & & & \\
\hline $\mathrm{Rb}$ & & 262.2 & 170.1 & 189.3 & 157 & 159 & 172 & 241 & 136 \\
\hline Sr & & 294.1 & 295.5 & 348.5 & 223 & 216 & 208 & 192 & 174 \\
\hline Y & & 31.7 & 21.0 & 36.7 & 23 & 19.4 & 26 & 37 & 28 \\
\hline
\end{tabular}


Regmi et al. (2016) Australian Journal of Earth Sciences, 63; http://dx.doi.org/10.1080/08120099.2016.1180321

\begin{tabular}{|c|c|c|c|c|c|c|c|c|}
\hline $\mathrm{Zr}$ & 177.1 & 138.9 & 201.9 & 158 & 130 & 187 & 182 & 167 \\
\hline $\mathrm{Nb}$ & 20.6 & 14.8 & 20.2 & 15.3 & 14.1 & 19.4 & 29 & 17.4 \\
\hline Mo & 3.9 & 1.1 & 3.5 & 0.8 & 0.8 & 1.7 & 0.5 & 0.5 \\
\hline $\mathrm{Cd}$ & 0.1 & 0.1 & 0.1 & & & & & \\
\hline Sn & 2.8 & 2.7 & 3.5 & 10.2 & 10.2 & 20 & 23 & 18.1 \\
\hline $\mathrm{Sb}$ & 0.3 & 0.6 & 0.2 & & & & & \\
\hline Cs & 13.9 & 10.3 & 11.2 & 7.8 & 8.7 & 13.1 & 19.9 & 11.5 \\
\hline $\mathrm{Ba}$ & 1191.0 & 806.0 & 908.9 & 714 & 795 & 718 & 825 & 391 \\
\hline La & 36.2 & 29.6 & 37.3 & 30 & 27 & 42 & 26 & 16.1 \\
\hline $\mathrm{Ce}$ & 79.7 & 51.7 & 66.4 & 58 & 52 & 84 & 68 & 42 \\
\hline $\operatorname{Pr}$ & 8.5 & 5.8 & 8.2 & 6.4 & 5.8 & 9.2 & 9.2 & 5.8 \\
\hline $\mathrm{Nd}$ & 31.2 & 20.2 & 30.1 & 23 & 20 & 32 & 37 & 23 \\
\hline Sm & 6.5 & 4.0 & 6.3 & 4.4 & 3.9 & 5.9 & 8.2 & 5.1 \\
\hline $\mathrm{Eu}$ & 1.4 & 1.1 & 1.5 & 1.1 & 1.0 & 1.5 & 1.4 & 1.1 \\
\hline $\mathrm{Gd}$ & 5.6 & 3.6 & 6.0 & 4.1 & 3.6 & 5.4 & 7.5 & 4.8 \\
\hline $\mathrm{Tb}$ & 0.9 & 0.6 & 1.0 & 0.6 & 0.5 & 0.8 & 1.2 & 0.8 \\
\hline Dy & 5.4 & 3.4 & 6.1 & 3.6 & 3.2 & 4.4 & 6.8 & 4.5 \\
\hline Ho & 1.1 & 0.7 & 1.3 & 0.7 & 0.6 & 0.9 & 1.4 & 0.9 \\
\hline $\mathrm{Er}$ & 3.1 & 2.0 & 3.5 & 2.1 & 1.9 & 2.5 & 4.1 & 2.7 \\
\hline Tm & 0.4 & 0.3 & 0.5 & 0.3 & 0.3 & 0.4 & 0.6 & 0.4 \\
\hline $\mathrm{Yb}$ & 2.8 & 1.9 & 3.1 & 2.1 & 1.8 & 2.3 & 4.0 & 2.7 \\
\hline Lu & 0.4 & 0.3 & 0.5 & 0.3 & 0.3 & 0.3 & 0.6 & 0.4 \\
\hline $\mathrm{Hf}$ & 4.8 & 3.7 & 5.2 & 4.0 & 3.4 & 4.1 & 4.1 & 4.1 \\
\hline $\mathrm{Ta}$ & 3.5 & 2.6 & 2.4 & 1.4 & 1.2 & 1.3 & 2.3 & 1.1 \\
\hline TI & 1.4 & 0.9 & 1.1 & & & & & \\
\hline $\mathrm{Pb}$ & 24.2 & 23.2 & 19.2 & 20 & 22 & 19.0 & 17.6 & 16.3 \\
\hline Th & 24.0 & 18.9 & 18.9 & 17.5 & 20 & 11.6 & 6.8 & 6.0 \\
\hline U & 6.8 & 5.1 & 4.4 & 2.6 & 2.1 & 1.4 & 3.0 & 2.1 \\
\hline
\end{tabular}




\begin{tabular}{|c|c|c|c|c|c|c|c|c|c|c|c|}
\hline $\begin{array}{c}\text { Pluton } \\
\text { Rock type }\end{array}$ & $\begin{array}{c}\text { Tanjil } \\
\text { Granite }\end{array}$ & $\begin{array}{l}\text { Tanjil } \\
\text { Granite }\end{array}$ & $\begin{array}{c}\text { Tanjil } \\
\text { Granite }\end{array}$ & $\begin{array}{l}\text { Tanjil } \\
\text { Granite }\end{array}$ & $\begin{array}{c}\text { Tanjil } \\
\text { Granite }\end{array}$ & $\begin{array}{c}\text { Tanjil } \\
\text { Granite }\end{array}$ & $\begin{array}{c}\text { Baw Baw } \\
\text { Granodiorite }\end{array}$ & $\begin{array}{c}\text { Baw Baw } \\
\text { Granodiorite }\end{array}$ & $\begin{array}{c}\text { Baw Baw } \\
\text { Granodiorite }\end{array}$ & $\begin{array}{c}\text { Baw Baw } \\
\text { Granodiorite }\end{array}$ & $\begin{array}{r}\text { Bi } \\
\text { Bi } \\
\text { Gra } \\
\text { iol }\end{array}$ \\
\hline Sample No & TANJ1 & 301 & 302 & 303 & 313 & 314 & $304-1$ & $305-2$ & 306 & 307 & $\mathrm{~B} /$ \\
\hline $\mathrm{SiO}_{2}$ & 70.29 & 72.25 & 72.34 & 69.99 & 72.14 & 71.56 & 67.29 & 66.99 & 66.47 & 67.16 & 6 \\
\hline $\mathrm{TiO}_{2}$ & 0.4 & 0.27 & 0.29 & 0.49 & 0.32 & 0.33 & 0.72 & 0.71 & 0.74 & 0.7 & 1 \\
\hline $\mathrm{Al}_{2} \mathrm{O}_{3}$ & 14.4 & 13.64 & 13.89 & 14.38 & 13.83 & 14.23 & 14.67 & 14.67 & 14.75 & 14.63 & 1. \\
\hline $\mathrm{Fe}_{2} \mathrm{O}_{3}$ & 2.98 & 2.05 & 2.05 & 3.18 & 2.21 & 2.25 & 4.14 & 4.35 & 4.5 & 4.25 & . \\
\hline $\mathrm{MnO}$ & 0.06 & 0.05 & 0.05 & 0.06 & 0.05 & 0.05 & 0.07 & 0.07 & 0.08 & 0.06 & । \\
\hline $\mathrm{MgO}$ & 0.95 & 0.6 & 0.62 & 1.21 & 0.69 & 0.7 & 1.77 & 1.89 & 1.92 & 1.85 & \\
\hline $\mathrm{CaO}$ & 2.7 & 1.99 & 2.02 & 2.77 & 2.13 & 2.38 & 3.38 & 3.33 & 2.55 & 3.25 & : \\
\hline $\mathrm{Na}_{2} \mathrm{O}$ & 3.87 & 3.57 & 3.62 & 3.62 & 3.6 & 3.83 & 3.14 & 3.4 & 2.96 & 3.34 & : \\
\hline $\mathrm{K}_{2} \mathrm{O}$ & 3.07 & 3.71 & 3.62 & 3.24 & 3.56 & 3.36 & 3.67 & 3.21 & 3.34 & 3.27 & : \\
\hline $\mathrm{P}_{2} \mathrm{O}_{5}$ & 0.12 & 0.06 & 0.06 & 0.11 & 0.07 & 0.07 & 0.14 & 0.14 & 0.14 & 0.14 & । \\
\hline L.O.I. & 0.96 & 0.87 & 1.05 & 0.97 & 0.67 & 0.72 & 0.54 & 0.75 & 1.81 & 0.58 & \\
\hline Total & 99.8 & 99.09 & 99.61 & 100.01 & 99.27 & 99.49 & 99.56 & 99.54 & 99.25 & 99.25 & 9 \\
\hline ASI & 0.985 & 1.009 & 1.024 & 0.991 & 1.012 & 0.996 & 0.959 & 0.969 & 1.123 & 0.978 & 0 \\
\hline $\mathrm{Li}$ & 60.8 & 57 & & 51 & & & 42 & & 32 & & \\
\hline $\mathrm{Be}$ & 2.9 & & & & & & & & & & \\
\hline Sc & 6.2 & 3.7 & & 6.7 & & & 9.2 & & 7.8 & & \\
\hline V & 37.0 & 26 & & 49 & & & 70 & & 59 & & I \\
\hline $\mathrm{Cr}$ & 11.0 & 27 & & 31 & & & 105 & & 62 & & : \\
\hline Co & 92.3 & 32 & & 46 & & & 21 & & 23 & & : \\
\hline $\mathrm{Ni}$ & 7.3 & 5.1 & & 15.8 & & & 23 & & 23 & & : \\
\hline $\mathrm{Cu}$ & 13.6 & 110 & & 48 & & & 27 & & 30 & & \\
\hline $\mathrm{Zn}$ & 48.5 & 37 & & 56 & & & 58 & & 52 & & I \\
\hline $\mathrm{Ga}$ & 16.3 & 14.2 & & 15.3 & & & 16.0 & & 13.6 & & \\
\hline ASI & 1.1 & & & & & & & & & & \\
\hline $\mathrm{Rb}$ & 158.2 & 172 & & 135 & & & 137 & & 109 & & 11 \\
\hline $\mathrm{Sr}$ & 229.9 & 180 & & 204 & & & 243 & & 162 & & 2 \\
\hline
\end{tabular}


Regmi et al. (2016) Australian Journal of Earth Sciences, 63; http://dx.doi.org/10.1080/08120099.2016.1180321

\begin{tabular}{|c|c|c|c|c|c|c|}
\hline$Y$ & 28.7 & 25 & 33 & 29 & 23 & : \\
\hline $\mathrm{Zr}$ & 148.8 & 134 & 177 & 212 & 147 & $1 !$ \\
\hline $\mathrm{Nb}$ & 15.2 & 12.5 & 14.8 & 13.5 & 10.0 & \\
\hline Mo & 1.4 & 0.3 & 1.2 & 1.6 & 0.3 & \\
\hline $\mathrm{Cd}$ & 0.1 & & & & & \\
\hline Sn & 4.0 & 4.4 & 5.0 & 3.5 & 3.0 & \\
\hline $\mathrm{Sb}$ & 0.2 & & & & & \\
\hline Cs & 8.0 & 10.6 & 8.5 & 8.1 & 4.8 & \\
\hline $\mathrm{Ba}$ & 718.6 & 747 & 888 & 1541 & 1054 & 11 \\
\hline La & 34.2 & 28 & 35 & 28 & 18.9 & : \\
\hline $\mathrm{Ce}$ & 64.6 & 51 & 65 & 53 & 37 & : \\
\hline $\mathrm{Pr}$ & 6.9 & 5.2 & 6.9 & 6.0 & 4.5 & \\
\hline $\mathrm{Nd}$ & 23.8 & 18 & 25 & 23 & 17.7 & : \\
\hline Sm & 4.8 & 4.0 & 5.5 & 5.4 & 4.6 & \\
\hline Eu & 0.9 & 0.7 & 1.0 & 1.5 & 1.2 & \\
\hline $\mathrm{Gd}$ & 4.5 & 3.6 & 5.1 & 5.0 & 4.1 & \\
\hline $\mathrm{Tb}$ & 0.7 & 0.7 & 0.9 & 0.9 & 0.7 & \\
\hline Dy & 4.5 & 4.1 & 5.6 & 5.2 & 4.4 & \\
\hline Ho & 1.0 & 0.8 & 1.1 & 1.0 & 0.9 & \\
\hline $\mathrm{Er}$ & 2.7 & 2.5 & 3.3 & 2.9 & 2.5 & \\
\hline Tm & 0.4 & 0.4 & 0.5 & 0.4 & 0.3 & \\
\hline $\mathrm{Yb}$ & 2.7 & 2.4 & 3.2 & 2.6 & 2.3 & \\
\hline Lu & 0.4 & 0.4 & 0.5 & 0.4 & 0.3 & \\
\hline $\mathrm{Hf}$ & 4.0 & 4.1 & 5.2 & 5.7 & 4.0 & \\
\hline $\mathrm{Ta}$ & 2.7 & 1.5 & 1.8 & 1.2 & 0.9 & \\
\hline TI & 0.8 & & & & & \\
\hline $\mathrm{Pb}$ & 17.4 & 21.1 & 17.5 & 20.2 & 15.0 & \\
\hline Th & 17.8 & 14.6 & 15.8 & 11.3 & 7.5 & \\
\hline$U$ & 3.7 & 5.7 & 4.6 & 3.6 & 2.0 & \\
\hline
\end{tabular}




\begin{tabular}{|c|c|c|c|c|c|c|c|c|c|}
\hline $\begin{array}{c}\text { Pluton } \\
\text { Rock type }\end{array}$ & $\begin{array}{c}\text { Baw Baw } \\
\text { Granodiorite }\end{array}$ & $\begin{array}{c}\text { Baw Baw } \\
\text { Granodiorite }\end{array}$ & $\begin{array}{c}\text { Baw Baw } \\
\text { Granodiorite }\end{array}$ & $\begin{array}{l}\text { Baw Baw } \\
\text { Enclave }\end{array}$ & $\begin{array}{l}\text { Baw Baw } \\
\text { Enclave }\end{array}$ & $\begin{array}{c}\text { Toorongo } \\
\text { Granodiorite }\end{array}$ & $\begin{array}{c}\text { Toorongo } \\
\text { Granodiorite }\end{array}$ & $\begin{array}{c}\text { Toorongo } \\
\text { Granodiorite }\end{array}$ & $\begin{array}{c}\text { Toorongo } \\
\text { Granodiorite }\end{array}$ \\
\hline Sample No & BAW1 & BAW2 & 311 & $304-1-1$ & $305-1$ & 402 & 408 & TOO1 & 315 \\
\hline $\mathrm{SiO}_{2}$ & 67.65 & 66.92 & 67.74 & 55.45 & 60.58 & 66.23 & 64.38 & 64.41 & 66.14 \\
\hline $\mathrm{TiO}_{2}$ & 0.69 & 0.69 & 0.72 & 1.04 & 0.93 & 0.78 & 0.85 & 0.85 & 0.62 \\
\hline $\mathrm{Al}_{2} \mathrm{O}_{3}$ & 14.71 & 14.98 & 14.74 & 13.58 & 14.57 & 15.07 & 15.72 & 15.36 & 16.39 \\
\hline $\mathrm{Fe}_{2} \mathrm{O}_{3}$ & 4.17 & 4.28 & 4.18 & 9.07 & 7.19 & 4.61 & 5.13 & 5.18 & 3.58 \\
\hline $\mathrm{MnO}$ & 0.06 & 0.06 & 0.06 & 0.23 & 0.14 & 0.06 & 0.08 & 0.08 & 0.06 \\
\hline $\mathrm{MgO}$ & 1.84 & 1.86 & 1.72 & 5.24 & 3.9 & 1.99 & 2.29 & 2.49 & 1.37 \\
\hline $\mathrm{CaO}$ & 3.19 & 3.34 & 3.31 & 6.5 & 5.23 & 3.44 & 4.43 & 4.16 & 4.11 \\
\hline $\mathrm{Na}_{2} \mathrm{O}$ & 3.22 & 3.33 & 3.31 & 2.64 & 3.69 & 3.47 & 3.7 & 3.36 & 4.03 \\
\hline $\mathrm{K}_{2} \mathrm{O}$ & 3.41 & 3.29 & 3.36 & 4.08 & 2.08 & 2.97 & 2.37 & 2.63 & 2.47 \\
\hline $\mathrm{P}_{2} \mathrm{O}_{5}$ & 0.18 & 0.19 & 0.14 & 0.18 & 0.21 & 0.16 & 0.17 & 0.2 & 0.13 \\
\hline L.O.I. & 0.7 & 0.68 & 0.57 & 0.94 & 0.91 & 0.54 & 0.54 & 0.87 & 0.48 \\
\hline Total & 99.86 & 99.65 & 99.85 & 98.98 & 99.43 & 99.32 & 99.68 & 99.63 & 99.39 \\
\hline ASI & 0.993 & 0.990 & 0.975 & 0.659 & 0.816 & 0.992 & 0.940 & 0.962 & 0.976 \\
\hline $\mathrm{Li}$ & 42.0 & 40.2 & 46 & 51 & 39 & 34.8 & 37.4 & 34.8 & 24 \\
\hline $\mathrm{Be}$ & 2.6 & 2.8 & & & & 2.5 & 2.4 & 2.4 & \\
\hline $\mathrm{Sc}$ & 10.6 & 10.9 & 9.3 & 24 & 25 & 10.9 & 12.7 & 13.2 & 4.9 \\
\hline V & 64.5 & 62.2 & 73 & 160 & 110 & 67.2 & 82.5 & 89.6 & 61 \\
\hline $\mathrm{Cr}$ & 52.2 & 53.2 & 94 & 305 & 164 & 56.7 & 73.7 & 76.3 & 60 \\
\hline Co & 81.8 & 92.6 & 29 & 32 & 27 & 30.9 & 19.6 & 94.1 & 19 \\
\hline $\mathrm{Ni}$ & 23.8 & 24.7 & 25 & 41 & 58 & 25.4 & 22.2 & 35.1 & 13.3 \\
\hline $\mathrm{Cu}$ & 19.4 & 23.7 & 30 & 21 & 17.1 & 19.9 & 34.1 & 38.6 & 25 \\
\hline $\mathrm{Zn}$ & 64.6 & 62.7 & 64 & 123 & 83 & 62.8 & 65.6 & 77.0 & 41 \\
\hline $\mathrm{Ga}$ & 18.3 & 18.5 & 16.7 & 16.1 & 15.1 & 18.5 & 18.7 & 19.6 & 15.7 \\
\hline ASI & 22.4 & 11.7 & & & & 1.3 & 1.4 & 1.2 & \\
\hline $\mathrm{Rb}$ & 162.0 & 153.6 & 136 & 164 & 96 & 144.2 & 107.9 & 127.9 & 37 \\
\hline $\mathrm{Sr}$ & 212.7 & 226.0 & 232 & 183 & 159 & 239.0 & 300.1 & 274.0 & 209 \\
\hline
\end{tabular}




\begin{tabular}{|c|c|c|c|c|c|c|c|c|c|}
\hline$Y$ & 38.1 & 39.1 & 35 & 67 & 61 & 35.4 & 32.3 & 31.1 & 20 \\
\hline $\mathrm{Zr}$ & 199.7 & 215.5 & 262 & 143 & 155 & 211.7 & 216.6 & 222.4 & 130 \\
\hline $\mathrm{Nb}$ & 14.5 & 15.0 & 14.3 & 16.3 & 14.4 & 15.1 & 13.2 & 14.7 & 10.8 \\
\hline Mo & 2.1 & 2.3 & 1.7 & 1.6 & 0.9 & 1.5 & 2.0 & 3.0 & 1.7 \\
\hline $\mathrm{Cd}$ & 0.1 & 0.1 & & & & 0.1 & 0.1 & 0.1 & \\
\hline Sn & 4.3 & 5.1 & 3.8 & 6.5 & 4.2 & 3.3 & 3.4 & 2.9 & 2.0 \\
\hline $\mathrm{Sb}$ & 0.4 & 0.3 & & & & 0.2 & 0.5 & 0.3 & \\
\hline Cs & 7.8 & 9.4 & 6.9 & 7.2 & 7.2 & 6.8 & 7.0 & 6.2 & 3.2 \\
\hline $\mathrm{Ba}$ & 1162.5 & 1077.9 & 1089 & 1504 & 644 & 1177.8 & 1015.6 & 1215.5 & 878 \\
\hline $\mathrm{La}$ & 29.0 & 38.2 & 39 & 14.3 & 18.6 & 40.3 & 26.6 & 27.4 & 14.5 \\
\hline $\mathrm{Ce}$ & 59.6 & 70.5 & 75 & 37 & 48 & 77.5 & 55.1 & 55.8 & 32 \\
\hline $\operatorname{Pr}$ & 7.1 & 8.9 & 8.3 & 5.8 & 6.9 & 9.4 & 6.6 & 6.6 & 3.4 \\
\hline $\mathrm{Nd}$ & 27.3 & 32.8 & 30 & & 31 & 34.0 & 26.1 & 25.7 & 13.1 \\
\hline Sm & 6.4 & 7.0 & 6.7 & 10.0 & 9.1 & 6.9 & 5.9 & 5.9 & 3.3 \\
\hline $\mathrm{Eu}$ & 1.3 & 1.4 & 1.4 & 1.3 & 1.0 & 1.5 & 1.6 & 1.6 & 1.2 \\
\hline $\mathrm{Gd}$ & 6.1 & 6.5 & 6.0 & 10.2 & 8.8 & 6.2 & 5.6 & 5.5 & 3.2 \\
\hline $\mathrm{Tb}$ & 1.0 & 1.1 & 1.0 & 2.0 & 1.7 & 1.0 & 0.9 & 0.9 & 0.6 \\
\hline Dy & 6.3 & 6.5 & 6.2 & 12.5 & 10.6 & 6.0 & 5.5 & 5.3 & 3.6 \\
\hline Ho & 1.4 & 1.4 & 1.2 & 2.5 & 2.1 & 1.3 & 1.2 & 1.1 & 0.7 \\
\hline $\mathrm{Er}$ & 3.7 & 3.8 & 3.5 & 6.9 & 6.2 & 3.4 & 3.2 & 3.1 & 2.2 \\
\hline $\mathrm{Tm}$ & 0.5 & 0.5 & 0.5 & 0.9 & 0.9 & 0.5 & 0.4 & 0.4 & 0.3 \\
\hline $\mathrm{Yb}$ & 3.3 & 3.5 & 3.2 & 6.1 & 5.9 & 3.0 & 3.0 & 2.7 & 2.1 \\
\hline $\mathrm{Lu}$ & 0.5 & 0.5 & 0.5 & 0.9 & 0.9 & 0.4 & 0.4 & 0.4 & 0.3 \\
\hline $\mathrm{Hf}$ & 5.1 & 5.4 & 7.0 & 3.8 & 4.5 & 5.2 & 5.4 & 5.3 & 3.6 \\
\hline $\mathrm{Ta}$ & 2.3 & 2.6 & 1.2 & 1.0 & 1.3 & 1.2 & 1.1 & 2.3 & 1.1 \\
\hline TI & 1.0 & 0.9 & & & & 0.9 & 0.8 & 0.8 & \\
\hline $\mathrm{Pb}$ & 18.4 & 18.5 & 18.9 & 19.0 & 11.8 & 16.9 & 15.9 & 15.2 & 11.8 \\
\hline Th & 13.8 & 13.6 & 13.5 & 5.3 & 12.4 & 12.7 & 62.3 & 8.6 & 5.3 \\
\hline U & 3.5 & 3.7 & 3.8 & 2.2 & 2.6 & 2.9 & 11.3 & 2.4 & 2.7 \\
\hline
\end{tabular}




\begin{tabular}{|c|c|c|c|c|c|c|c|c|c|}
\hline Pluton & Toorongo & Toorongo & Tynong & Tynong & Tynong & Tynong & Tynong & Tynong & Toorongo \\
\hline Rock type & Granodiorite & Granodiorite & Granite & Granite & Granite & Granite & Granite & Granite & Granodiorite \\
\hline Sample No & 403 & 406 & 203 & $2802-2$ & 2805 & $2803-7$ & 2803-8 & $2803-5$ & 315 \\
\hline $\mathrm{SiO}_{2}$ & 64.18 & 63.32 & 67.85 & 65.98 & 69.75 & 70.88 & 67.99 & 63.36 & 66.14 \\
\hline $\mathrm{TiO}_{2}$ & 0.85 & 0.91 & 0.55 & 0.54 & 0.46 & 0.34 & 0.48 & 0.38 & 0.62 \\
\hline $\mathrm{Al}_{2} \mathrm{O}_{3}$ & 15.95 & 15.82 & 15.53 & 14.5 & 14.65 & 14.26 & 15.18 & 18.32 & 16.39 \\
\hline $\mathrm{Fe}_{2} \mathrm{O}_{3}$ & 5.16 & 5.37 & 3.41 & 4.31 & 2.78 & 1.99 & 3.12 & 2.5 & 3.58 \\
\hline $\mathrm{MnO}$ & 0.08 & 0.09 & 0.05 & 0.07 & 0.04 & 0.03 & 0.04 & 0.03 & 0.06 \\
\hline $\mathrm{MgO}$ & 2.31 & 2.57 & 1.26 & 2.43 & 0.93 & 0.77 & 1.13 & 1.02 & 1.37 \\
\hline $\mathrm{CaO}$ & 4.54 & 4.57 & 3.16 & 4.18 & 2.68 & 2.04 & 3.36 & 3.53 & 4.11 \\
\hline $\mathrm{Na}_{2} \mathrm{O}$ & 3.8 & 3.57 & 3.94 & 3.59 & 3.71 & 3.37 & 3.6 & 4.83 & 4.03 \\
\hline $\mathrm{K}_{2} \mathrm{O}$ & 2.32 & 2.33 & 3.22 & 1.94 & 3.46 & 4.42 & 2.66 & 3.22 & 2.47 \\
\hline $\mathrm{P}_{2} \mathrm{O}_{5}$ & 0.17 & 0.19 & 0.13 & 0.14 & 0.1 & 0.21 & 0.22 & 0.2 & 0.13 \\
\hline L.O.I. & 0.55 & 0.64 & 0.64 & 1.62 & 0.39 & 1.09 & 1.23 & 1.67 & 0.48 \\
\hline Total & 99.94 & 99.4 & 99.74 & 99.33 & 98.95 & 99.4 & 99.02 & 99.07 & 99.39 \\
\hline ASI & 0.936 & 0.946 & 0.987 & 0.928 & 0.994 & 1.015 & 1.017 & 1.025 & 0.976 \\
\hline $\mathrm{Li}$ & 32 & 45 & & 50 & & 49 & & 41 & 24 \\
\hline $\mathrm{Be}$ & & & & 2.3 & & 1.7 & & 3.0 & \\
\hline Sc & 11.4 & 11.9 & & 12.2 & & 6.9 & & 7.5 & 4.9 \\
\hline V & 91 & 106 & & 80 & & 29 & & 42 & 61 \\
\hline $\mathrm{Cr}$ & 66 & 116 & & 205 & & 75 & & 73 & 60 \\
\hline Co & 35 & 20 & & 16.5 & & 3.6 & & 7.2 & 19 \\
\hline $\mathrm{Ni}$ & 30 & 35 & & 108 & & 7.6 & & 11.1 & 13.3 \\
\hline $\mathrm{Cu}$ & 50 & 76 & & 648 & & 5.8 & & 5.4 & 25 \\
\hline $\mathrm{Zn}$ & 67 & 76 & & 71 & & 17.6 & & 25.4 & 41 \\
\hline $\mathrm{Ga}$ & 16.5 & 18.1 & & 16.5 & & 15.9 & & 21.1 & 15.7 \\
\hline \multicolumn{10}{|l|}{ ASI } \\
\hline $\mathrm{Rb}$ & 85 & 98 & & 89 & & 108 & & 143 & 37 \\
\hline $\mathrm{Sr}$ & 287 & 296 & & 235 & & 157 & & 296 & 209 \\
\hline$Y$ & 32 & 33 & & 26 & & 22 & & 27 & 20 \\
\hline
\end{tabular}


Regmi et al. (2016) Australian Journal of Earth Sciences, 63; http://dx.doi.org/10.1080/08120099.2016.1180321

\begin{tabular}{|c|c|c|c|c|c|c|}
\hline $\mathrm{Zr}$ & 206 & 218 & 293 & 193 & 135 & 130 \\
\hline $\mathrm{Nb}$ & 12.5 & 14.5 & 20.8 & 12.4 & 15.4 & 10.8 \\
\hline Mo & 1.3 & 1.8 & 0.8 & 4.2 & 137.7 & 1.7 \\
\hline \multicolumn{7}{|l|}{$\mathrm{Cd}$} \\
\hline Sn & 1.9 & 3.0 & 8.0 & 3.1 & 3.6 & 2.0 \\
\hline \multicolumn{7}{|l|}{$\mathrm{Sb}$} \\
\hline Cs & 4.0 & 5.4 & 7.7 & 12.5 & 9.5 & 3.2 \\
\hline $\mathrm{Ba}$ & 959 & 1059 & 598 & 973 & 477 & 878 \\
\hline $\mathrm{La}$ & 35 & 37 & 38 & 24 & 14.4 & 14.5 \\
\hline $\mathrm{Ce}$ & 67 & 68 & 73 & 58 & 29 & 32 \\
\hline $\mathrm{Pr}$ & 7.5 & 8.0 & 7.6 & 5.9 & 3.4 & 3.4 \\
\hline $\mathrm{Nd}$ & 28 & 29 & 24 & 19.1 & 11.7 & 13.1 \\
\hline $\mathrm{Sm}$ & 6.4 & 6.4 & 4.5 & 4.0 & 3.0 & 3.3 \\
\hline Eu & 1.5 & 1.6 & 1.2 & 0.9 & 1.3 & 1.2 \\
\hline $\mathrm{Gd}$ & 5.9 & 5.8 & 4.2 & 3.6 & 3.1 & 3.2 \\
\hline $\mathrm{Tb}$ & 1.0 & 1.0 & 0.7 & 0.7 & 0.7 & 0.6 \\
\hline Dy & 6.0 & 5.9 & 4.1 & 3.9 & 4.1 & 3.6 \\
\hline Ho & 1.2 & 1.1 & 0.9 & 0.9 & 0.9 & 0.7 \\
\hline $\mathrm{Er}$ & 3.3 & 3.3 & 2.5 & 2.3 & 2.6 & 2.2 \\
\hline Tm & 0.4 & 0.5 & 0.4 & 0.4 & 0.4 & 0.3 \\
\hline $\mathrm{Yb}$ & 2.8 & 3.0 & 2.5 & 2.2 & 2.8 & 2.1 \\
\hline Lu & 0.4 & 0.5 & 0.4 & 0.3 & 0.4 & 0.3 \\
\hline $\mathrm{Hf}$ & 5.3 & 5.7 & 7.7 & 5.4 & 4.1 & 3.6 \\
\hline $\mathrm{Ta}$ & 0.9 & 1.4 & 1.6 & 1.1 & 1.9 & 1.1 \\
\hline \multicolumn{7}{|l|}{ TI } \\
\hline $\mathrm{Pb}$ & 11.6 & 13.7 & 12.3 & 30 & 26 & 11.8 \\
\hline Th & 8.9 & 9.4 & 14.4 & 16.2 & 4.9 & 5.3 \\
\hline$U$ & 2.1 & 3.2 & 4.0 & 3.0 & 6.6 & 2.7 \\
\hline
\end{tabular}




\begin{tabular}{|c|c|c|c|c|c|c|c|c|c|}
\hline $\begin{array}{c}\text { Pluton } \\
\text { Rock type }\end{array}$ & $\begin{array}{c}\text { Tynong } \\
\text { Porphyritic } \\
\text { granite } \\
\end{array}$ & $\begin{array}{c}\text { Tynong } \\
\text { Porphyritic } \\
\text { granite } \\
\end{array}$ & $\begin{array}{l}\text { Tynong } \\
\text { Aplitic } \\
\text { dyke }\end{array}$ & $\begin{array}{c}\text { Tynong } \\
\text { Granodiorite }\end{array}$ & $\begin{array}{c}\text { Tynong } \\
\text { Granodiorite }\end{array}$ & $\begin{array}{l}\text { Tynong } \\
\text { Granite }\end{array}$ & $\begin{array}{l}\text { Tynong } \\
\text { Granite }\end{array}$ & $\begin{array}{c}\text { Tynong } \\
\text { Granodiorite }\end{array}$ & $\begin{array}{c}\text { Tynong } \\
\text { Granodiorite }\end{array}$ \\
\hline Sample No & $2803-6$ & 120204 & 120207 & 120205 & 120206 & 120202 & $2803-1$ & TYN1A & TYN1B \\
\hline $\mathrm{SiO}_{2}$ & 65.2 & 66.49 & 76.61 & 75.41 & 76.02 & 72.18 & 71.12 & 69.38 & 72.19 \\
\hline $\mathrm{TiO}_{2}$ & 0.37 & 0.52 & 0.1 & 0.22 & 0.18 & 0.49 & 0.35 & 0.45 & 0.37 \\
\hline $\mathrm{Al}_{2} \mathrm{O}_{3}$ & 17.75 & 15.73 & 12.18 & 12.92 & 12.32 & 12.73 & 14.31 & 15.01 & 13.34 \\
\hline $\mathrm{Fe}_{2} \mathrm{O}_{3}$ & 2.27 & 3.54 & 0.94 & 0.96 & 1.2 & 3.43 & 2.33 & 2.95 & 2.65 \\
\hline $\mathrm{MnO}$ & 0.04 & 0.06 & 0.04 & 0.01 & 0.02 & 0.07 & 0.04 & 0.05 & 0.05 \\
\hline $\mathrm{MgO}$ & 0.9 & 1.29 & 0.12 & 0.25 & 0.59 & 1.23 & 0.73 & 0.94 & 0.99 \\
\hline $\mathrm{CaO}$ & 3.48 & 3.34 & 0.47 & 0.74 & 0.59 & 1.67 & 2.55 & 2.58 & 2.2 \\
\hline $\mathrm{Na}_{2} \mathrm{O}$ & 4.76 & 4.11 & 3.07 & 3.32 & 2.87 & 3.43 & 3.63 & 3.89 & 3.56 \\
\hline $\mathrm{K}_{2} \mathrm{O}$ & 3.28 & 3.04 & 5.4 & 4.5 & 5.69 & 2.8 & 3.33 & 3.77 & 3.7 \\
\hline $\mathrm{P}_{2} \mathrm{O}_{5}$ & 0.19 & 0.11 & 0.01 & 0.01 & 0.03 & 0.12 & 0.09 & 0.11 & 0.1 \\
\hline L.O.I. & 1.42 & 0.98 & 0.47 & 0.69 & 0.34 & 0.88 & 0.71 & 0.74 & 0.65 \\
\hline Total & 99.66 & 99.23 & 99.42 & 99.03 & 99.88 & 99.02 & 99.2 & 99.88 & 99.81 \\
\hline ASI & 1.001 & 0.974 & 1.035 & 1.105 & 1.029 & 1.086 & 1.006 & 0.988 & 0.961 \\
\hline $\mathrm{Li}$ & 33 & & & 52.8 & 49.0 & 118.5 & 41.4 & 42.4 & 34.9 \\
\hline $\mathrm{Be}$ & 2.8 & & & 4.1 & 2.5 & 3.2 & 2.4 & 2.9 & 2.3 \\
\hline Sc & 4.9 & & & 3.7 & 3.3 & 7.1 & 4.0 & 6.6 & 5.6 \\
\hline V & 24 & & & 11.6 & 7.9 & 48.4 & 28.0 & 40.0 & 34.5 \\
\hline $\mathrm{Cr}$ & 82 & & & 76.2 & 85.6 & 111.9 & 64.4 & 16.6 & 18.0 \\
\hline Co & 5.6 & & & 3.2 & 1.4 & 7.4 & 4.1 & 84.7 & 98.2 \\
\hline $\mathrm{Ni}$ & 9.0 & & & 3.7 & 2.4 & 13.5 & 6.2 & 9.9 & 9.6 \\
\hline $\mathrm{Cu}$ & 4.7 & & & 27.4 & 0.9 & 96.2 & 17.9 & 69.1 & 32.7 \\
\hline $\mathrm{Zn}$ & 21.2 & & & 31.7 & 24.9 & 52.4 & 34.7 & 52.6 & 42.8 \\
\hline $\mathrm{Ga}$ & 18.5 & & & 15.7 & 14.5 & 15.6 & 15.2 & 18.2 & 14.3 \\
\hline ASI & & & & 3.8 & 0.1 & 3.7 & 0.9 & 1.3 & 0.5 \\
\hline $\mathrm{Rb}$ & 79 & & & 304.4 & 252.1 & 191.8 & 171.0 & 186.8 & 165.8 \\
\hline $\mathrm{Sr}$ & 252 & & & 39.8 & 22.6 & 113.5 & 194.5 & 189.3 & 132.1 \\
\hline
\end{tabular}




\begin{tabular}{|c|c|c|c|c|c|c|c|}
\hline Y & 17.8 & 56.2 & 47.0 & 39.9 & 28.1 & 42.8 & 34.3 \\
\hline $\mathrm{Zr}$ & 105 & 137.5 & 104.9 & 202.1 & 113.8 & 211.6 & 162.1 \\
\hline $\mathrm{Nb}$ & 8.5 & 16.9 & 15.4 & 19.0 & 12.6 & 16.8 & 14.1 \\
\hline Mo & 3.0 & 0.9 & 0.6 & 1.2 & 0.5 & 3.1 & 1.9 \\
\hline $\mathrm{Cd}$ & & 0.3 & 0.0 & 0.1 & 0.1 & 0.1 & 0.1 \\
\hline Sn & 2.8 & 9.5 & 3.6 & 4.7 & 4.1 & 4.5 & 4.8 \\
\hline $\mathrm{Sb}$ & & 0.9 & 0.3 & 2.5 & 0.4 & 0.4 & 0.3 \\
\hline Cs & 7.1 & 13.5 & 9.7 & 10.7 & 11.4 & 6.9 & 9.3 \\
\hline $\mathrm{Ba}$ & 465 & 405.3 & 266.0 & 492.1 & 772.4 & 1290.9 & 720.9 \\
\hline $\mathrm{La}$ & 8.9 & 20.4 & 36.6 & 40.1 & 38.5 & 53.0 & 41.2 \\
\hline $\mathrm{Ce}$ & 18.0 & 42.3 & 79.2 & 72.0 & 71.6 & 96.5 & 72.1 \\
\hline $\operatorname{Pr}$ & 2.1 & 4.8 & 9.4 & 8.4 & 7.8 & 10.5 & 8.4 \\
\hline $\mathrm{Nd}$ & 7.6 & 17.0 & 34.4 & 29.3 & 26.0 & 35.7 & 28.3 \\
\hline Sm & 2.0 & 4.3 & 8.6 & 6.2 & 5.1 & 7.2 & 5.7 \\
\hline $\mathrm{Eu}$ & 1.1 & 0.3 & 0.2 & 0.6 & 0.9 & 1.1 & 0.8 \\
\hline $\mathrm{Gd}$ & 2.1 & 4.6 & 7.0 & 5.7 & 4.4 & 6.6 & 5.3 \\
\hline $\mathrm{Tb}$ & 0.5 & 1.0 & 1.2 & 1.0 & 0.7 & 1.1 & 0.9 \\
\hline Dy & 2.9 & 7.1 & 7.3 & 6.2 & 4.3 & 6.8 & 5.6 \\
\hline Ho & 0.7 & 1.7 & 1.6 & 1.4 & 0.9 & 1.5 & 1.2 \\
\hline $\mathrm{Er}$ & 1.8 & 5.3 & 4.6 & 3.8 & 2.6 & 4.2 & 3.5 \\
\hline $\mathrm{Tm}$ & 0.3 & 0.8 & 0.7 & 0.6 & 0.4 & 0.6 & 0.5 \\
\hline $\mathrm{Yb}$ & 2.0 & 5.8 & 4.8 & 3.8 & 2.6 & 4.0 & 3.5 \\
\hline Lu & 0.3 & 0.9 & 0.7 & 0.6 & 0.4 & 0.6 & 0.5 \\
\hline $\mathrm{Hf}$ & 3.1 & 4.7 & 3.8 & 5.3 & 3.1 & 5.7 & 4.6 \\
\hline $\mathrm{Ta}$ & 0.9 & 2.6 & 1.6 & 2.0 & 1.4 & 2.8 & 2.7 \\
\hline TI & & 1.8 & 1.6 & 1.1 & 1.0 & 1.1 & 1.1 \\
\hline $\mathrm{Pb}$ & 28 & 33.3 & 30.1 & 18.0 & 19.9 & 20.1 & 19.5 \\
\hline Th & 3.1 & 44.9 & 41.8 & 26.0 & 13.9 & 18.3 & 21.8 \\
\hline U & 4.8 & 15.5 & 11.9 & 6.9 & 5.1 & 6.3 & 5.6 \\
\hline
\end{tabular}




\begin{tabular}{|c|c|c|c|c|c|c|c|c|c|}
\hline Pluton & Tynong & Tynong & Tynong & Tynong & Tynong & Tynong & Tynong & Tynong & Tynong \\
\hline Rock type & Granodiorite & Granodiorite & Granodiorite & Granodiorite & Granodiorite & Enclave & Diorite & Diorite & Enclave \\
\hline Sample No & TYN3 & TYN4 & TYN5 & TYN6 & TYN7 & TYN1C-B & 2803-10 & 2810 & $2803-4$ \\
\hline $\mathrm{SiO}_{2}$ & 72.55 & 73.94 & 77.38 & 70.26 & 67.35 & 57.49 & 55.29 & 57.46 & 58.34 \\
\hline $\mathrm{TiO}_{2}$ & 0.39 & 0.11 & 0.27 & 0.43 & 0.61 & 1.21 & 1.33 & 1.25 & 1.18 \\
\hline $\mathrm{Al}_{2} \mathrm{O}_{3}$ & 13.26 & 14.39 & 11.45 & 14.47 & 15.5 & 16.48 & 16.62 & 16.43 & 16.34 \\
\hline $\mathrm{Fe}_{2} \mathrm{O}_{3}$ & 2.58 & 0.91 & 1.68 & 2.57 & 3.78 & 7.85 & 8.17 & 7.73 & 7.44 \\
\hline $\mathrm{MnO}$ & 0.04 & 0.02 & 0.02 & 0.04 & 0.05 & 0.13 & 0.13 & 0.13 & 0.13 \\
\hline $\mathrm{MgO}$ & 0.71 & 0.17 & 0.43 & 0.72 & 1.19 & 3.59 & 3.33 & 3.31 & 2.94 \\
\hline $\mathrm{CaO}$ & 2.07 & 2.07 & 1.13 & 2.11 & 3.4 & 6.39 & 6.65 & 6.39 & 6.33 \\
\hline $\mathrm{Na}_{2} \mathrm{O}$ & 3.27 & 3.27 & 2.55 & 3.69 & 3.82 & 3.13 & 3.22 & 3.29 & 3.24 \\
\hline $\mathrm{K}_{2} \mathrm{O}$ & 3.8 & 4.77 & 4.56 & 3.6 & 3.18 & 1.88 & 1.76 & 1.57 & 1.54 \\
\hline $\mathrm{P}_{2} \mathrm{O}_{5}$ & 0.11 & 0.03 & 0.08 & 0.12 & 0.16 & 0.43 & 0.46 & 0.41 & 0.41 \\
\hline L.O.I. & 1 & 0.38 & 0.36 & 1.51 & 0.68 & 1.17 & 2.48 & 1.15 & 1.38 \\
\hline Total & 99.78 & 100.07 & 99.93 & 99.63 & 99.73 & 99.77 & 99.43 & 99.11 & 99.28 \\
\hline ASI & 0.999 & 1.004 & 1.022 & 1.047 & 0.973 & 0.875 & 0.860 & 0.876 & 0.882 \\
\hline $\mathrm{Li}$ & 55.5 & 46.9 & 35.0 & 54.6 & 40.2 & 58.5 & 71 & 64 & 41 \\
\hline $\mathrm{Be}$ & 2.9 & 3.4 & 2.1 & 2.9 & 2.8 & 2.0 & 1.7 & 2.3 & 1.9 \\
\hline Sc & 5.9 & 2.6 & 4.6 & 6.6 & 8.9 & 16.7 & 17.4 & 21 & 16.8 \\
\hline V & 29.7 & 6.6 & 18.3 & 29.5 & 57.0 & 176.5 & 138 & 178 & 130 \\
\hline $\mathrm{Cr}$ & 12.6 & 3.0 & 8.8 & 12.6 & 16.9 & 25.2 & 64 & 83 & 73 \\
\hline Co & 70.9 & 91.5 & 137.3 & 116.7 & 93.1 & 84.9 & 22 & 26 & 19.6 \\
\hline $\mathrm{Ni}$ & 6.6 & 1.7 & 4.7 & 8.3 & 9.6 & 18.5 & 20 & 24 & 19.9 \\
\hline $\mathrm{Cu}$ & 10.9 & 4.0 & 5.0 & 77.0 & 15.7 & 81.0 & 83 & 83 & 74 \\
\hline $\mathrm{Zn}$ & 38.2 & 20.9 & 31.5 & 47.2 & 55.4 & 95.1 & 90 & 110 & 90 \\
\hline $\mathrm{Ga}$ & 16.2 & 16.1 & 13.7 & 17.4 & 19.3 & 18.1 & 16.0 & 21.0 & 17.0 \\
\hline ASI & 0.0 & 0.6 & 0.4 & 63.8 & 1.3 & 0.4 & & & \\
\hline $\mathrm{Rb}$ & 183.0 & 210.9 & 202.2 & 182.4 & 140.6 & 80.1 & 106 & 73 & 47 \\
\hline $\mathrm{Sr}$ & 148.1 & 126.8 & 94.8 & 144.3 & 227.0 & 395.1 & 380 & 448 & 393 \\
\hline$Y$ & 41.5 & 32.8 & 37.1 & 57.0 & 36.9 & 34.1 & 26 & 34 & 29 \\
\hline
\end{tabular}


Regmi et al. (2016) Australian Journal of Earth Sciences, 63; $h$ ttp://dx.doi.org/10.1080/08120099.2016.1180321

\begin{tabular}{|c|c|c|c|c|c|c|c|c|c|}
\hline $\mathrm{Zr}$ & 195.1 & 66.1 & 100.7 & 171.6 & 217.0 & 214.2 & 236 & 200 & 221 \\
\hline $\mathrm{Nb}$ & 13.1 & 7.1 & 8.5 & 15.4 & 15.1 & 23.8 & 21 & 30 & 26 \\
\hline Mo & 1.8 & 1.3 & 2.1 & 2.3 & 2.4 & 1.9 & 5.3 & 1.4 & 1.5 \\
\hline $\mathrm{Cd}$ & 0.1 & 0.1 & 0.0 & 0.2 & 0.1 & 0.1 & & & \\
\hline Sn & 3.8 & 3.2 & 5.2 & 7.8 & 3.8 & 3.4 & 5.0 & 1.9 & 1.6 \\
\hline $\mathrm{Sb}$ & 0.1 & 0.4 & 0.1 & 0.4 & 0.4 & 0.3 & & & \\
\hline Cs & 13.4 & 10.3 & 7.7 & 8.3 & 6.5 & 10.2 & 11.4 & 9.5 & 4.5 \\
\hline $\mathrm{Ba}$ & 748.7 & 957.1 & 918.2 & 439.6 & 1243.0 & 517.3 & 588 & 641 & 533 \\
\hline La & 41.2 & 22.7 & 33.2 & 63.5 & 54.5 & 34.8 & 30 & 43 & 36 \\
\hline $\mathrm{Ce}$ & 74.6 & 45.2 & 68.4 & 106.7 & 98.8 & 70.7 & 62 & 88 & 76 \\
\hline $\mathrm{Pr}$ & 9.1 & 5.1 & 7.7 & 12.8 & 11.0 & 7.9 & 7.0 & 9.6 & 8.2 \\
\hline $\mathrm{Nd}$ & 31.8 & 18.1 & 27.2 & 44.9 & 38.1 & 28.7 & 23 & 32 & 28 \\
\hline $\mathrm{Sm}$ & 6.7 & 4.3 & 5.9 & 9.3 & 7.4 & 5.9 & 4.9 & 6.4 & 5.5 \\
\hline Eu & 0.9 & 0.8 & 0.7 & 1.1 & 1.5 & 1.5 & 1.5 & 2.1 & 1.8 \\
\hline $\mathrm{Gd}$ & 6.2 & 4.2 & 5.6 & 8.9 & 6.7 & 5.6 & 4.5 & 6.0 & 5.2 \\
\hline $\mathrm{Tb}$ & 1.1 & 0.8 & 1.0 & 1.5 & 1.1 & 0.9 & 0.8 & 1.0 & 0.9 \\
\hline Dy & 6.8 & 5.2 & 6.0 & 9.1 & 6.4 & 5.7 & 4.4 & 5.7 & 4.9 \\
\hline $\mathrm{Ho}$ & 1.5 & 1.2 & 1.3 & 2.0 & 1.4 & 1.3 & 1.0 & 1.2 & 1.1 \\
\hline $\mathrm{Er}$ & 4.1 & 3.3 & 3.6 & 5.3 & 3.7 & 3.4 & 2.6 & 3.3 & 2.8 \\
\hline Tm & 0.6 & 0.5 & 0.5 & 0.7 & 0.5 & 0.5 & 0.4 & 0.5 & 0.4 \\
\hline $\mathrm{Yb}$ & 3.8 & 3.3 & 3.1 & 4.6 & 3.3 & 3.2 & 2.5 & 3.1 & 2.7 \\
\hline Lu & 0.6 & 0.5 & 0.4 & 0.7 & 0.5 & 0.5 & 0.4 & 0.5 & 0.4 \\
\hline $\mathrm{Hf}$ & 5.2 & 2.2 & 2.8 & 4.6 & 5.6 & 5.1 & 5.5 & 5.3 & 5.2 \\
\hline $\mathrm{Ta}$ & 2.2 & 1.9 & 1.8 & 2.6 & 2.5 & 2.4 & 1.1 & 1.7 & 1.4 \\
\hline $\mathrm{TI}$ & 1.1 & 1.3 & 1.2 & 1.7 & 0.8 & 0.8 & & & \\
\hline $\mathrm{Pb}$ & 18.3 & 28.7 & 23.5 & 23.7 & 16.0 & 8.2 & 10.9 & 12.9 & 10.3 \\
\hline Th & 19.7 & 14.5 & 17.3 & 23.5 & 16.8 & 5.2 & 4.6 & 8.2 & 7.5 \\
\hline$U$ & 4.1 & 4.5 & 4.0 & 5.3 & 2.6 & 1.5 & 1.9 & 2.3 & 2.7 \\
\hline
\end{tabular}




\begin{tabular}{|c|c|c|c|}
\hline Pluton & Tynong & Tynong & Tynong \\
\hline Rock type & Diorite & $\begin{array}{l}\text { Hybrid diorite } \\
\text { (ocelli) }\end{array}$ & $\begin{array}{l}\text { Hybrid diorite } \\
\text { (ocelli) }\end{array}$ \\
\hline Sample No & $2803-2$ & 2801 & $2802-3$ \\
\hline $\mathrm{SiO}_{2}$ & 57.81 & 61.38 & 58.52 \\
\hline $\mathrm{TiO}_{2}$ & 1.24 & 0.87 & 0.65 \\
\hline $\mathrm{Al}_{2} \mathrm{O}_{3}$ & 16.45 & 14.27 & 14.36 \\
\hline $\mathrm{Fe}_{2} \mathrm{O}_{3}$ & 7.59 & 6.28 & 6.33 \\
\hline $\mathrm{MnO}$ & 0.13 & 0.12 & 0.12 \\
\hline $\mathrm{MgO}$ & 3.03 & 4.02 & 4.87 \\
\hline $\mathrm{CaO}$ & 6.11 & 5.21 & 6.34 \\
\hline $\mathrm{Na}_{2} \mathrm{O}$ & 3.25 & 3.28 & 2.84 \\
\hline $\mathrm{K}_{2} \mathrm{O}$ & 1.83 & 2.48 & 1.75 \\
\hline $\mathrm{P}_{2} \mathrm{O}_{5}$ & 0.42 & 0.26 & 0.19 \\
\hline L.O.I. & 1.15 & 1.7 & 3.01 \\
\hline Total & 99.02 & 99.9 & 98.99 \\
\hline ASI & 0.891 & 0.812 & 0.793 \\
\hline $\mathrm{Li}$ & 52 & 49 & 74 \\
\hline $\mathrm{Be}$ & 2.0 & 1.8 & 1.7 \\
\hline $\mathrm{Sc}$ & 17.8 & 16.9 & 20 \\
\hline V & 136 & 130 & 127 \\
\hline $\mathrm{Cr}$ & 73 & 244 & 341 \\
\hline Co & 21 & 25 & 33 \\
\hline $\mathrm{Ni}$ & 20 & 163 & 248 \\
\hline $\mathrm{Cu}$ & 124 & 116 & 112 \\
\hline $\mathrm{Zn}$ & 96 & 65 & 85 \\
\hline $\mathrm{Ga}$ & 17.9 & 15.1 & 15.2 \\
\hline \multicolumn{4}{|l|}{ ASI } \\
\hline $\mathrm{Rb}$ & 80 & 109 & 107 \\
\hline $\mathrm{Sr}$ & 390 & 232 & 263 \\
\hline Y & 33 & 30 & 27 \\
\hline
\end{tabular}


Regmi et al. (2016) Australian Journal of Earth Sciences, 63; http://dx.doi.org/10.1080/08120099.2016.1180321

\begin{tabular}{lrrr}
$\mathrm{Zr}$ & 351 & 216 & 229 \\
$\mathrm{Nb}$ & 26 & 23 & 17.6 \\
$\mathrm{Mo}$ & 2.3 & 5.2 & 2.6 \\
$\mathrm{Cd}$ & & & \\
$\mathrm{Sn}$ & 4.7 & 5.2 & 8.0 \\
$\mathrm{Sb}$ & & & \\
$\mathrm{Cs}$ & 11.5 & 9.6 & 10.4 \\
$\mathrm{Ba}$ & 594 & 686 & 532 \\
$\mathrm{La}$ & 38 & 36 & 28 \\
$\mathrm{Ce}$ & 80 & 76 & 59 \\
$\mathrm{Pr}$ & 8.9 & 8.5 & 6.8 \\
$\mathrm{Nd}$ & 30 & 28 & 23 \\
$\mathrm{Sm}$ & 6.0 & 5.4 & 4.8 \\
$\mathrm{Eu}$ & 1.8 & 1.4 & 1.4 \\
$\mathrm{Gd}$ & 5.6 & 5.0 & 4.5 \\
$\mathrm{~Tb}$ & 1.0 & 0.9 & 0.8 \\
$\mathrm{Dy}$ & 5.4 & 4.8 & 4.4 \\
$\mathrm{Ho}$ & 1.2 & 1.0 & 1.0 \\
$\mathrm{Er}$ & 3.2 & 2.8 & 2.6 \\
$\mathrm{Tm}$ & 0.5 & 0.4 & 0.4 \\
$\mathrm{Yb}$ & 3.1 & 2.7 & 2.5 \\
$\mathrm{Lu}$ & 0.5 & 0.4 & 0.4 \\
$\mathrm{Hf}$ & 8.0 & 5.4 & 5.6 \\
$\mathrm{Ta}$ & 1.4 & 1.5 & 1.1 \\
$\mathrm{Tl}$ & & & \\
$\mathrm{Pb}$ & 11.1 & 10.1 & 7.5 \\
$\mathrm{Th}$ & 5.3 & 7.6 & 5.9 \\
$\mathrm{U}$ & 1.6 & 3.2 & 2.0 \\
\hline
\end{tabular}


Table $4 \mathrm{Lu}$ and $\mathrm{Hf}$ isotopic data for zircons from one sample of the Toorongo (sample 406, 37 $48^{\prime} 41^{\prime \prime} \mathrm{S}, 146^{\circ} 05^{\prime} 40^{\prime \prime} \mathrm{E}$ ), and two samples from the Tynong pluton (samples 2803-8 and TYN5, 38 $\left.03^{\prime} 30^{\prime \prime} \mathrm{S}, 145^{\circ} 37^{\prime} 26^{\prime \prime} \mathrm{E}\right)$ ), Lachlan Orogen, eastern Australia.

\begin{tabular}{|c|c|c|c|c|c|c|c|c|c|c|c|c|c|}
\hline $\begin{array}{l}\text { Sample } \\
\text { sample }\end{array}$ & $\begin{array}{l}\text { Grain/ } \\
\text { spot \# }\end{array}$ & $\begin{array}{c}\text { Total Hf, } \\
\text { V }\end{array}$ & ${ }^{176} \mathrm{Lu} /{ }^{177} \mathrm{Hf}$ & $\pm 2 S E$ & ${ }^{176} \mathrm{Hf} /{ }^{177} \mathrm{Hf}$ & $\pm 2 S E$ & ${ }^{178} \mathrm{Hf} /{ }^{177} \mathrm{Hf}$ & $\varepsilon \mathrm{Hf}(0)$ & $\begin{array}{c}\text { Age, } \\
\mathrm{Ma}\end{array}$ & ${ }^{176} \mathrm{Hf} /{ }^{177} \mathrm{Hf}(\mathrm{t})$ & $\varepsilon \mathrm{Hf}(\mathrm{t})$ & $\pm 2 S E$ & $\begin{array}{c}\mathrm{T}_{\mathrm{DM} 2}, \\
\mathrm{Ga}\end{array}$ \\
\hline $\begin{array}{l}\text { sample } \\
406\end{array}$ & 4 & 8.7 & 0.00146 & 0.00001 & 0.282644 & 0.000054 & 1.4675 & -4.5 & 370 & 0.282634 & 3.2 & 1.9 & 1.08 \\
\hline \multirow{2}{*}{\multicolumn{14}{|c|}{$\begin{array}{l}\text { Toorongo } \\
\text { Grd }\end{array}$}} \\
\hline & 5 & 7.0 & 0.00145 & 0.00005 & 0.282722 & 0.000054 & 1.4676 & -1.8 & 370 & 0.282712 & 6.0 & 1.9 & 0.91 \\
\hline & 6 & 8.7 & 0.00095 & 0.00002 & 0.282660 & 0.000050 & 1.4675 & -4.0 & 370 & 0.282653 & 3.9 & 1.8 & 1.04 \\
\hline & 8 & 7.6 & 0.00146 & 0.00001 & 0.282617 & 0.000060 & 1.4674 & -5.5 & 370 & 0.282607 & 2.3 & 2.1 & 1.14 \\
\hline & 10 & 8.4 & 0.00118 & 0.00001 & 0.282649 & 0.000049 & 1.4674 & -4.3 & 370 & 0.282641 & 3.5 & 1.7 & 1.07 \\
\hline & 12 & 7.6 & 0.00127 & 0.00001 & 0.282655 & 0.000053 & 1.4674 & -4.1 & 370 & 0.282646 & 3.7 & 1.9 & 1.06 \\
\hline & 13 & 10.0 & 0.00100 & 0.00002 & 0.282519 & 0.000046 & 1.4674 & -8.9 & 370 & 0.282512 & -1.1 & 1.6 & 1.35 \\
\hline & 14 & 6.8 & 0.00326 & 0.00003 & 0.282785 & 0.000062 & 1.4674 & 0.5 & 370 & 0.282762 & 7.8 & 2.2 & 0.80 \\
\hline & 15 & 8.6 & 0.00123 & 0.00001 & 0.282578 & 0.000052 & 1.4674 & -6.9 & 370 & 0.282570 & 1.0 & 1.8 & 1.22 \\
\hline & 17 & 8.5 & 0.00122 & 0.00002 & 0.282638 & 0.000055 & 1.4675 & -4.7 & 370 & 0.282630 & 3.1 & 1.9 & 1.09 \\
\hline & 18 & 7.6 & 0.00180 & 0.00005 & 0.282726 & 0.000055 & 1.4675 & -1.6 & 370 & 0.282714 & 6.1 & 1.9 & 0.91 \\
\hline & 22 & 7.0 & 0.00139 & 0.00004 & 0.282646 & 0.000059 & 1.4674 & -4.5 & 370 & 0.282636 & 3.3 & 2.1 & 1.08 \\
\hline & 24 & 8.6 & 0.00209 & 0.00001 & 0.282665 & 0.000053 & 1.4675 & -3.8 & 370 & 0.282651 & 3.8 & 1.9 & 1.05 \\
\hline & 31 & 9.0 & 0.00099 & 0.00001 & 0.282729 & 0.000048 & 1.4675 & -1.5 & 370 & 0.282722 & 6.4 & 1.7 & 0.89 \\
\hline & 35 & 7.3 & 0.00179 & 0.00002 & 0.282686 & 0.000059 & 1.4675 & -3.0 & 370 & 0.282674 & 4.7 & 2.1 & 1.00 \\
\hline & 38 & 6.2 & 0.00145 & 0.00001 & 0.282660 & 0.000062 & 1.4674 & -4.0 & 370 & 0.282650 & 3.8 & 2.2 & 1.05 \\
\hline & 44 & 8.8 & 0.00108 & 0.00002 & 0.282670 & 0.000053 & 1.4675 & -3.6 & 370 & 0.282663 & 4.3 & 1.9 & 1.02 \\
\hline \multicolumn{14}{|l|}{ sample } \\
\hline $\begin{array}{l}2803-8 \\
\text { Tynong }\end{array}$ & 2 & 9.2 & 0.00157 & 0.00006 & 0.282801 & 0.000048 & 1.4675 & 1.0 & 370 & 0.282790 & 8.8 & 1.7 & 0.74 \\
\hline \multirow[t]{8}{*}{ Grd. } & 3 & 7.9 & 0.00227 & 0.00003 & 0.282769 & 0.000053 & 1.4674 & -0.1 & 370 & 0.282753 & 7.5 & 1.9 & 0.82 \\
\hline & 4 & 9.7 & 0.00125 & 0.00001 & 0.282732 & 0.000055 & 1.4675 & -1.4 & 370 & 0.282723 & 6.4 & 1.9 & 0.89 \\
\hline & 6 & 10.4 & 0.00087 & 0.00003 & 0.282720 & 0.000051 & 1.4675 & -1.8 & 370 & 0.282714 & 6.1 & 1.8 & 0.91 \\
\hline & 7 & 10.2 & 0.00131 & 0.00002 & 0.282678 & 0.000048 & 1.4675 & -3.3 & 370 & 0.282669 & 4.5 & 1.7 & 1.01 \\
\hline & 8 & 8.4 & 0.00195 & 0.00003 & 0.282741 & 0.000054 & 1.4675 & -1.1 & 370 & 0.282728 & 6.6 & 1.9 & 0.88 \\
\hline & 10 & 9.1 & 0.00160 & 0.00005 & 0.282729 & 0.000048 & 1.4674 & -1.5 & 370 & 0.282718 & 6.2 & 1.7 & 0.90 \\
\hline & 11 & 9.4 & 0.00167 & 0.00002 & 0.282783 & 0.000049 & 1.4675 & 0.4 & 370 & 0.282771 & 8.1 & 1.7 & 0.78 \\
\hline & 13 & 7.1 & 0.00167 & 0.00002 & 0.282777 & 0.000054 & 1.4675 & 0.2 & 370 & 0.282765 & 7.9 & 1.9 & 0.80 \\
\hline
\end{tabular}




\begin{tabular}{|c|c|c|c|c|c|c|c|c|c|c|c|c|}
\hline 14 & 10.7 & 0.00114 & 0.00002 & 0.282740 & 0.000048 & 1.4675 & -1.1 & 370 & 0.282732 & 6.7 & 1.7 & 0.87 \\
\hline 16 & 9.9 & 0.00121 & 0.00003 & 0.282752 & 0.000046 & 1.4675 & -0.7 & 370 & 0.282744 & 7.1 & 1.6 & 0.84 \\
\hline 17 & 11.1 & 0.00186 & 0.00004 & 0.282775 & 0.000053 & 1.4676 & 0.1 & 370 & 0.282762 & 7.8 & 1.9 & 0.80 \\
\hline 18 & 8.4 & 0.00094 & 0.00002 & 0.282760 & 0.000048 & 1.4675 & -0.4 & 370 & 0.282754 & 7.5 & 1.7 & 0.82 \\
\hline 20 & 9.7 & 0.00172 & 0.00005 & 0.282749 & 0.000054 & 1.4675 & -0.8 & 370 & 0.282737 & 6.9 & 1.9 & 0.86 \\
\hline 21 & 9.4 & 0.00130 & 0.00003 & 0.282717 & 0.000051 & 1.4675 & -1.9 & 370 & 0.282708 & 5.9 & 1.8 & 0.92 \\
\hline 22 & 7.1 & 0.00159 & 0.00003 & 0.282794 & 0.000062 & 1.4676 & 0.8 & 370 & 0.282783 & 8.5 & 2.2 & 0.76 \\
\hline 25 & 8.4 & 0.00116 & 0.00003 & 0.282703 & 0.000048 & 1.4675 & -2.4 & 370 & 0.282695 & 5.4 & 1.7 & 0.95 \\
\hline 26 & 7.8 & 0.00255 & 0.00014 & 0.282783 & 0.000064 & 1.4675 & 0.4 & 370 & 0.282765 & 7.9 & 2.2 & 0.80 \\
\hline 27 & 10.2 & 0.00134 & 0.00003 & 0.282738 & 0.000048 & 1.4675 & -1.2 & 370 & 0.282729 & 6.6 & 1.7 & 0.88 \\
\hline 32 & 9.1 & 0.00156 & 0.00003 & 0.282764 & 0.000060 & 1.4675 & -0.3 & 370 & 0.282753 & 7.5 & 2.1 & 0.82 \\
\hline 33 & 9.5 & 0.00133 & 0.00001 & 0.282738 & 0.000050 & 1.4676 & -1.2 & 370 & 0.282729 & 6.6 & 1.8 & 0.88 \\
\hline 34 & 8.5 & 0.00120 & 0.00002 & 0.282761 & 0.000052 & 1.4675 & -0.4 & 370 & 0.282753 & 7.5 & 1.8 & 0.82 \\
\hline 36 & 10.0 & 0.00090 & 0.00001 & 0.282671 & 0.000050 & 1.4675 & -3.6 & 370 & 0.282665 & 4.3 & 1.8 & 1.02 \\
\hline 1 & 5.7 & 0.00133 & 0.00003 & 0.282704 & 0.000094 & 1.4676 & -2.4 & 370 & 0.282695 & 5.4 & 3.3 & 0.95 \\
\hline 2 & 4.2 & 0.00154 & 0.00001 & 0.282710 & 0.000110 & 1.4677 & -2.2 & 370 & 0.282699 & 5.6 & 3.9 & 0.94 \\
\hline 3 & 4.2 & 0.00171 & 0.00003 & 0.282692 & 0.000094 & 1.4675 & -2.8 & 370 & 0.282680 & 4.9 & 3.3 & 0.98 \\
\hline 4.1 & 5.3 & 0.00174 & 0.00002 & 0.282702 & 0.000088 & 1.4675 & -2.5 & 370 & 0.282690 & 5.2 & 3.1 & 0.96 \\
\hline 4.2 & 5.7 & 0.00153 & 0.00002 & 0.282651 & 0.000080 & 1.4676 & -4.3 & 370 & 0.282640 & 3.5 & 2.8 & 1.07 \\
\hline 5 & 4.6 & 0.00129 & 0.00001 & 0.282662 & 0.000091 & 1.4676 & -3.9 & 370 & 0.282653 & 3.9 & 3.2 & 1.04 \\
\hline 6 & 4.6 & 0.00394 & 0.00004 & 0.282641 & 0.000099 & 1.4675 & -4.6 & 370 & 0.282614 & 2.5 & 3.5 & 1.13 \\
\hline 7.1 & 5.9 & 0.00190 & 0.00001 & 0.282705 & 0.000076 & 1.4677 & -2.4 & 370 & 0.282692 & 5.3 & 2.7 & 0.96 \\
\hline 7.2 & 4.9 & 0.00196 & 0.00001 & 0.282583 & 0.000082 & 1.4675 & -6.7 & 370 & 0.282569 & 1.0 & 2.9 & 1.22 \\
\hline 8 & 4.4 & 0.00150 & 0.00003 & 0.282677 & 0.000099 & 1.4676 & -3.4 & 370 & 0.282667 & 4.4 & 3.5 & 1.01 \\
\hline 9 & 3.3 & 0.00698 & 0.00016 & 0.282770 & 0.000150 & 1.4675 & -0.1 & 370 & 0.282722 & 6.4 & 5.3 & 0.89 \\
\hline 10 & 4.9 & 0.00199 & 0.00005 & 0.282657 & 0.000079 & 1.4676 & -4.1 & 370 & 0.282643 & 3.6 & 2.8 & 1.06 \\
\hline 11.2 & 4.0 & 0.00147 & 0.00002 & 0.282610 & 0.000100 & 1.4675 & -5.7 & 370 & 0.282600 & 2.0 & 3.5 & 1.16 \\
\hline 11.1 & 4.7 & 0.00125 & 0.00001 & 0.282680 & 0.000100 & 1.4675 & -3.3 & 370 & 0.282671 & 4.6 & 3.5 & 1.00 \\
\hline 12 & 3.9 & 0.00284 & 0.00003 & 0.282780 & 0.000110 & 1.4677 & 0.3 & 370 & 0.282760 & 7.7 & 3.9 & 0.81 \\
\hline 13 & 4.1 & 0.00155 & 0.00002 & 0.282690 & 0.000110 & 1.4677 & -2.9 & 370 & 0.282679 & 4.9 & 3.9 & 0.98 \\
\hline 14 & 4.2 & 0.00173 & 0.00003 & 0.282656 & 0.000092 & 1.4676 & -4.1 & 370 & 0.282644 & 3.6 & 3.3 & 1.06 \\
\hline
\end{tabular}

$\begin{array}{lcc}\begin{array}{l}\text { sample } \\ \text { TYN5 } \\ \text { Tynong }\end{array} & 1 & 5.7 \\ \text { Grd. } & 2 & 4.2 \\ & 3 & 4.2 \\ & 4.1 & 5.3 \\ & 4.2 & 5.7 \\ & 5 & 4.6 \\ & 6 & 4.6 \\ & 7.1 & 5.9 \\ & 7.2 & 4.9 \\ & 8 & 4.4 \\ & 9 & 3.3 \\ & 10 & 4.9 \\ & 11.2 & 4.0 \\ & 11.1 & 4.7 \\ & 12 & 3.9 \\ & 13 & 4.1 \\ & 14 & 4.2\end{array}$

0.00173

0.00003

$0.282656 \quad 0.000092$

1.4676 


\begin{tabular}{ccccccccccccc}
15.1 & 4.1 & 0.00243 & 0.00006 & 0.282700 & 0.000110 & 1.4676 & -2.5 & 370 & 0.282683 & 5.0 & 3.9 & 0.98 \\
15.2 & 4.1 & 0.00254 & 0.00009 & 0.282790 & 0.000120 & 1.4676 & 0.6 & 370 & 0.282772 & 8.2 & 4.2 & 0.78 \\
16 & 3.9 & 0.00120 & 0.00001 & 0.282690 & 0.000110 & 1.4676 & -2.9 & 370 & 0.282682 & 4.9 & 3.9 & 0.98 \\
17 & 3.7 & 0.00184 & 0.00004 & 0.282620 & 0.000120 & 1.4676 & -5.4 & 370 & 0.282607 & 2.3 & 4.2 & 1.14 \\
18 & 4.5 & 0.00221 & 0.00006 & 0.282710 & 0.000100 & 1.4677 & -2.2 & 370 & 0.282695 & 5.4 & 3.5 & 0.95 \\
19 & 3.2 & 0.00245 & 0.00003 & 0.282620 & 0.000130 & 1.4676 & -5.4 & 370 & 0.282603 & 2.2 & 4.6 & 1.15 \\
20.1 & 3.9 & 0.00149 & 0.00001 & 0.282630 & 0.000110 & 1.4676 & -5.0 & 370 & 0.282620 & 2.7 & 3.9 & 1.12 \\
20.2 & 4.3 & 0.00296 & 0.00009 & 0.282690 & 0.000100 & 1.4676 & -2.9 & 370 & 0.282669 & 4.5 & 3.5 & 1.01 \\
21 & 3.9 & 0.00288 & 0.00014 & 0.282680 & 0.000110 & 1.4676 & -3.3 & 370 & 0.282660 & 4.2 & 3.9 & 1.03 \\
22 & 3.7 & 0.00165 & 0.00001 & 0.282530 & 0.000110 & 1.4675 & -8.6 & 370 & 0.282519 & -0.8 & 3.9 & 1.34 \\
23 & 3.9 & 0.00133 & 0.00004 & 0.282600 & 0.000120 & 1.4676 & -6.1 & 370 & 0.282591 & 1.7 & 4.2 & 1.18 \\
24.1 & 2.5 & 0.00452 & 0.00004 & 0.282640 & 0.000150 & 1.4676 & -4.7 & 370 & 0.282609 & 2.4 & 5.3 & 1.14 \\
24.2 & 2.0 & 0.00501 & 0.00007 & 0.282840 & 0.000180 & 1.4676 & 2.4 & 370 & 0.282805 & 9.3 & 6.4 & 0.71 \\
25 & 3.6 & 0.00110 & 0.00001 & 0.282730 & 0.000110 & 1.4677 & -1.5 & 370 & 0.282722 & 6.4 & 3.9 & 0.89 \\
26 & 3.5 & 0.00201 & 0.00002 & 0.282690 & 0.000120 & 1.4675 & -2.9 & 370 & 0.282676 & 4.7 & 4.2 & 0.99 \\
27 & 3.8 & 0.00169 & 0.00007 & 0.282720 & 0.000110 & 1.4677 & -1.8 & 370 & 0.282708 & 5.9 & 3.9 & 0.92 \\
28 & 3.9 & 0.00132 & 0.00001 & 0.282620 & 0.000100 & 1.4675 & -5.4 & 370 & 0.282611 & 2.4 & 3.5 & 1.13 \\
\hline
\end{tabular}

Notes Internal precision (2se) for measured ${ }^{176} \mathrm{Hf} /{ }^{177} \mathrm{Hf}$ scales inversely with total $\mathrm{Hf}$ signal during ablation, and increases sharply for signals $<6 \mathrm{~V} \mathrm{Hf}$. Initial $\mathrm{Hf}$ isotope compositions $\left({ }^{176} \mathrm{Hf} /{ }^{177} \mathrm{Hf}(\mathrm{t}), \varepsilon_{\mathrm{Hf}}(\mathrm{t})\right)$ calculated at $370 \mathrm{Ma}$, with 2 se errors in initial $\mathrm{e}_{\mathrm{Hf}}$ approximated by internal (2se) precision for measured (presentday) ${ }^{176} \mathrm{Hf} /{ }^{177} \mathrm{Hf}$ which are very close to the uncertainties obtained by propagating the errors in measured isotope and Lu/Hf ratios, and age (assumed here to be $\pm 5 \mathrm{Ma})$ 
Regmi et al. (2016) Australian Journal of Earth Sciences, 63; http://dx.doi.org/10.1080/08120099.2016.1180321

Results for standard zircons obtained in the course of this work

\begin{tabular}{|c|c|c|c|c|c|c|c|}
\hline $01500(1065$ & & ${ }^{176} \mathrm{Lu} /{ }^{177} \mathrm{Hf}$ & $2 s d$ & ${ }^{176} \mathrm{Hf} /{ }^{177} \mathrm{Hf}$ & $2 \mathrm{sd}$ & $\mathrm{e}_{\mathrm{Hf}}$ & $2 s d$ \\
\hline \multicolumn{8}{|c|}{91500 (1065 Ma) } \\
\hline $16 / 12 / 2011$ & $n-7$ & & י & & & -10.1 & 1.0 \\
\hline reference & $n=r$ & $\mathbf{0 . 0 0 0 3 1}$ & 0.00002 & 0.282306 & $\begin{array}{l}0.000135 \\
\mathbf{0 . 0 0 0 0 0 8}\end{array}$ & $\begin{array}{l}-15.6 \\
-16.5\end{array}$ & 4.8 \\
\hline \multicolumn{8}{|c|}{ BR266 (560 Ma) } \\
\hline $\begin{array}{l}24 / 03 / 2011 \\
\text { reference }\end{array}$ & $n=8$ & $\begin{array}{l}0.00022 \\
\mathbf{0 . 0 0 0 2 2}\end{array}$ & 0.00001 & $\begin{array}{l}0.281651 \\
\mathbf{0 . 2 8 1 6 3 0}\end{array}$ & $\begin{array}{c}0.000057 \\
\mathbf{0 . 0 0 0 0 1}\end{array}$ & $\begin{array}{l}-39.6 \\
-40.4\end{array}$ & 2.0 \\
\hline \multicolumn{8}{|c|}{ Temora-2 (417 Ma) } \\
\hline $\begin{array}{l}16 / 12 / 2011 \\
\text { reference }\end{array}$ & $\mathrm{n}=11$ & $\begin{array}{l}0.00117 \\
\mathbf{0 . 0 0 1 0 9}\end{array}$ & 0.00062 & $\begin{array}{l}0.282681 \\
\mathbf{0 . 2 8 2 6 8 6}\end{array}$ & $\begin{array}{l}0.000070 \\
\mathbf{0 . 0 0 0 0 0 8}\end{array}$ & $\begin{array}{l}-3.2 \\
-3.0\end{array}$ & 2.5 \\
\hline \multicolumn{8}{|c|}{ Plesovice (337 Ma) } \\
\hline $\begin{array}{l}16 / 12 / 2011 \\
\text { reference }\end{array}$ & $n=5$ & $\begin{array}{l}0.00012 \\
\mathbf{0 . 0 0 0 1 3}\end{array}$ & 0.00002 & $\begin{array}{l}0.282496 \\
\mathbf{0 . 2 8 2 4 8 2}\end{array}$ & $\begin{array}{l}0.000027 \\
\mathbf{0 . 0 0 0 0 1 3}\end{array}$ & $\begin{array}{c}-9.8 \\
-10.3\end{array}$ & 1.0 \\
\hline
\end{tabular}

Zircon reference values from Woodhead \& Hergt (2005) and Slama et al (2008) 